\title{
The energy transport by the propagation of sound waves in wave guides with a moving medium
}

\author{
P. LE GRAND \\ Department of Applied Mathematics, Twente Institute of Technology, Enschede, The Netherlands
}

(Received November 2, 1976)

\section{SUMMARY}

The problem of the propagation of sound waves radiated by a source in a fluid moving with subsonic velocity between two parallel walls or inside a cylindrical tube is considered in [2]. The most interesting thing of this problem is that waves may occur with constant amplitude coming from infinity. This article gives the calculation of the energy transport in the wave guides.

It is shown that it is not possible to gain energy from infinity.

\section{Introduction}

Reference [1] gives the investigation of an acoustical problem in a subsonic flow. In the solution of that problem incoming waves with constant amplitude occurred. The radiation condition of Sommerfeld says that, if the medium at infinity is homogeneous, no incoming waves can occur. This implies that the radiation condition of Sommerfeld is not applicable in the problem treated in [1] because the medium at infinity is not homogeneous. The incoming waves were a motive for Hoogstraten and Kaper to investigate two problems which have especially to do with these waves. In [2] they consider first the two-dimensional problem of a line source which is located in a subsonic flow between two parallel plates, and then they investigate the problem of a point source in a subsonic flow inside a circular cylindrical tube. The present article gives additional calculations about the energy transport. There is an energy transport by the various waves but there is also a transport of internal and kinetical energy by the main flow. It is shown that it is not possible to gain energy from infinity. This means that, if the Sommerfeld radiation condition is formulated as the requirement that an inflow of energy from infinity cannot occur, this condition is applicable in the problems treated here.

\section{The formulae for the velocity potential}

We shall use the same notation as in [2]. In the first problem two parallel walls coincide with the planes $\bar{y}= \pm d$ of a three-dimensional $\bar{x}, \bar{y}, \bar{z}$ space. Between the walls a non-viscous fluid moves with constant velocity $U$ in the direction of the positive $\bar{x}$-axis. The fluid velocity is subsonic, hence $U / c=M<1$, where $c$ is the sound velocity. An harmonically fluctuating line source with frequency $\omega$ is situated along the $\bar{z}$-axis. In the second problem we have a tube with radius $d$ and axis coinciding with the $\bar{x}$-axis. Here we have a point source in the origin. In both problems the source is switched on at time $\bar{t}=0$. We are interested in the solution for $\bar{t} \rightarrow \infty$. As is mentioned 
in [2] the steady state problem, that is, the limit problem for $\bar{t} \rightarrow \infty$, has not a unique solution. To obtain a unique solution one can introduce a bulk viscosity and then one can consider the limit when this viscosity tends to zero. This is done in [1] and [2]. A second possibility is to consider the complete initial value problem and to obtain from this the solution for $\bar{t} \rightarrow \infty$. This is also done in [2] and it is shown that both methods give the same results.

We shall not give again the derivations of the formulae. We want only to remark that the expressions in [2] become less complicated if one starts with an expansion in a Fourier series in the problem with the flat walls, and with a Dini series in the problem with the cylindrical tube. Hence for the dimensionless velocity potential in the first problem we put

$$
\Psi(x, y, t)=\frac{1}{2} V_{0}(x, t)+\sum_{n=1}^{\infty} V_{n}(x, t) \cos n \pi y,
$$

and for the second problem

$$
\Psi(x, r, t)=\sum_{n=0}^{\infty} W_{n}(x, t) J_{0}\left(\lambda_{n} r\right)
$$

Here we have used dimensionless quantities

$$
x=\bar{x} d^{-1}, \quad y=\bar{y} d^{-1}, \quad r=\bar{r} d^{-1}, \quad t=\omega \bar{t}, \quad \Psi=\bar{\Psi} \omega^{-1} d^{-2},
$$

where $\bar{\Psi}$ is the velocity potential and $\bar{r}$ is the radial coordinate. The numbers $\lambda_{n}$ are the zeros of the first-order Besselfunction, hence $J_{1}\left(\lambda_{n}\right)=0$ with $\lambda_{0}=0, \lambda_{n+1}>\lambda_{n}$.

The boundary condition of zero normal velocity at the walls of the wave guides is satisfied by each term of the series. The coefficients $V_{n}$ and $W_{n}$ can be found along the same way as is done in [2] for the potential $\Psi$.

For the initial value problem we obtain

$$
\begin{aligned}
& V_{n}(x, t)=\frac{1}{2 \pi} \int_{-\infty}^{\infty}\left\{\frac{-\mathrm{e}^{-i \lambda x}}{\beta^{2} \lambda^{2}+2 k M \lambda+n^{2} \pi^{2}-k^{2}} \mathrm{e}^{i t}\right. \\
& \left.\quad+\frac{\mathrm{e}^{-i \lambda x}}{2 k\left(s^{+}(\lambda)-1\right) \sqrt{\lambda^{2}+n^{2} \pi^{2}}} \mathrm{e}^{i s^{+}(\lambda) t}-\frac{\mathrm{e}^{-i \lambda x}}{2 k\left(s^{-}(\lambda)-1\right) \sqrt{\lambda^{2}+n^{2} \pi^{2}}} \mathrm{e}^{i s^{-}(\lambda) t}\right\} d \lambda
\end{aligned}
$$

where $k=\omega d / c, \beta=\sqrt{1-M^{2}}$ and $s^{ \pm}(\lambda)=M \lambda k^{-1} \pm k^{-1} \sqrt{\lambda^{2}+n^{2} \pi^{2}}$.

The solution (3) is unique because it can be shown that the integrand of (3) has no singularities on the real axis. A consequence of our series expansion(1) is that this can be proved easier than in [2]. A product expansion of the sine function is not necessary now.

For $t \rightarrow \infty$ and $n>0$ we get

$$
\begin{aligned}
& V_{n}(x, t)=\frac{-\mathrm{e}^{i t}}{2 \pi} \int_{L} \frac{\mathrm{e}^{-i \lambda x}}{\beta^{2} \lambda^{2}+2 k M \lambda+n^{2} \pi^{2}-k^{2}} d \lambda+\frac{1}{2 \pi} \sqrt{\frac{k}{2 n \beta}} \\
& \times\left\{\frac{\mathrm{e}^{i \pi\left[\frac{1}{4}+(n M / \beta) x+(n \beta / k) t\right]}}{n \pi \beta-k}+\frac{\mathrm{e}^{-i \pi\left[\frac{1}{4}+(n M / \beta) x+(n \beta / k) t\right]}}{n \pi \beta+k}\right\} \frac{1}{\sqrt{t}}\left(1+o\left(\frac{1}{t}\right)\right),
\end{aligned}
$$


and for $n=0$,

$$
V_{0}(x, t)=-\frac{\mathrm{e}^{i t}}{2 \pi} \int_{L} \frac{\mathrm{e}^{-i \lambda x}}{\beta^{2} \lambda^{2}+2 k M \lambda-k^{2}} d \lambda-\frac{i}{2 k} .
$$

The path of integration $L$ is the real axis, but for $n \leqq N$, where $N$ is the largest integer less than $k /(\beta \pi)$, this path has a small semi-circle below the point $\xi_{n}^{+}$and a small semi-circle above the point $\xi_{n}^{-}$, where

$$
\xi_{n}^{ \pm}=-\beta^{-2}\left(k M \pm \sqrt{k^{2}-\beta^{2} n^{2} \pi^{2}}\right), \quad n \leqq N .
$$

For $n>k /(\beta \pi)>N$ we define

$$
\xi_{n}^{ \pm}=\beta^{-2}\left(-k M \pm i \sqrt{\beta^{2} n^{2} \pi^{2}-k^{2}}\right), \quad n>N .
$$

The second term in (5) is not found when one uses the method of vanishing bulk viscosity. However this constant term has no practical meaning. It is possible to calculate the integrals in (4) and (5) by the method of residues.

If we neglect the second-order terms in (4) and (5) we obtain

$$
\begin{aligned}
V_{n}(x, t) & =\frac{i \mathrm{e}^{-i \xi_{n}^{ \pm} x}}{2 \sqrt{k^{2}-\beta^{2} n^{2} \pi^{2}}} \mathrm{e}^{i t}, \text { for } n \leqq N \\
& =\frac{\mathrm{e}^{-i \xi_{n}^{ \pm} x}}{2 \sqrt{\beta^{2} n^{2} \pi^{2}-k^{2}}} \mathrm{e}^{i t}, \text { for } n>N
\end{aligned}
$$

where we have to take $\xi_{n}^{-}$for $x>0$ and $\xi_{n}^{+}$for $x<0$. If there are numbers $\xi_{n}^{-}<0$ then there are downstream incoming waves with constant amplitude. This occurs if there exist integers $n$ with $k / \pi<n<k /(\beta \pi)$. The formulae for $W_{n}$ in the tube problem can be found from the expressions for $V_{n}$ by replacing everywhere $n$ by $\lambda_{n} / \pi$ and multiplying $V_{n}$ by $2 / J_{0}^{2}\left(\lambda_{n}\right)$.

\section{The energy transport}

We consider only the steady state solution. There is an energy transport by the waves. Especially the incoming waves may rise the question whether it is possible to gain downstream energy from infinity. However, we have to realise that internal and kinetical energy drifts downstream with the main flow. We shall show that it cannot occur, neither downstream nor upstream, that one gains energy from infinity.

First we consider the problem with the two parallel plates. We denote by $E_{T}$ the total energy that flows in the positive $x$-direction at (say) $x=x_{0}$ over a unit of length in the $z$-direction between the plates in a period of time $2 \pi$. Then $E_{T}=E_{1}+E_{2}$, where $E_{1}$ is the energy flow caused by the interaction of the fluid particles in the waves and $E_{2}$ is the flow of internal and kinetical energy. One can calculate $E_{1}$ by the principle that the increase of energy in a volume is equal to the work done by the external forces. Let $P_{\infty}$ be the pressure at infinity and $p$ the pressure disturbance caused by the source. The velocity of the main flow expressed in dimensional coordinates is $M / k$. The velocity disturbances in the $x$-direction are given by 
$\partial \Psi / \partial x$. Then we get

$$
\begin{aligned}
E_{1} & =-\int_{t=0}^{2 \pi} \int_{y=-1}^{1}\left(p_{\infty}+p\right)\left(M / k+\frac{\partial \Psi}{\partial x}\right) d y d t \\
& =-\int_{t=0}^{2 \pi} \int_{y=-1}^{1}\left(p_{\infty} M / k+p_{\infty} \frac{\partial \Psi}{\partial x}+p M / k+p \frac{\partial \Psi}{\partial x}\right) d t .
\end{aligned}
$$

Due to the periodic time dependence of $\Psi$ and $p$, the contribution of the second and third term in the integrand vanishes after integration over $t$. The first term does not depend on $t$ and $x$ and gives the energy flow of the undisturbed medium. We shall not consider this term. The pressure $p$ can be found from the equation of motion. In a coordinate system $x_{m}, y, t_{m}$ moving with the main flow we have $\operatorname{grad} p=p d q / d t$, where $\rho$ is the density and $q$ the velocity vector. Up to first order accuracy we have $d q / d t=\partial q / \partial t$. Then we find from the $x_{m}$-component of the equation of motion that $p=\rho\left(\partial \Psi / \partial t_{m}\right)$. The connection between the fixed and the moving coordinate system is

$$
x=x_{m}+M k^{-1} t_{m}, \quad t=t_{m} .
$$

This gives

$$
p=\rho\left(\frac{\partial \Psi}{\partial t}+\frac{M}{k} \frac{\partial \Psi}{\partial x}\right)
$$

and hence

$$
E_{1}=-\rho \int_{t=0}^{2 \pi} \int_{y=-1}^{1}\left\{\frac{\partial \Psi}{\partial t} \frac{\partial \Psi}{\partial x}+\frac{M}{k}\left(\frac{\partial \Psi}{\partial x}\right)^{2}\right\} d y d t
$$

For the calculation of $E_{2}$ we have to know the acoustical energy $E$, that is, the sum of the internal and kinetical energy per unit of volume. An expression for $E$ can be found in [3], formula (4.63), page 183. Expressed in our dimensionless quantities, this becomes

$$
E=\frac{\rho}{2}\left\{k^{2}\left(\frac{\partial \Psi}{\partial t_{m}}\right)^{2}+\left(\frac{\partial \Psi}{\partial x_{m}}\right)^{2}+\left(\frac{\partial \Psi}{\partial y}\right)^{2}+\left(\frac{\partial \Psi}{\partial z}\right)^{2}\right\} .
$$

As the velocity of the undisturbed flow is $M / k$, we obtain

$$
E_{2}=\frac{\rho M}{2 k} \int_{t=0}^{2 \pi} \int_{y=-1}^{1}\left\{k^{2}\left(\frac{\partial \Psi}{\partial t}+\frac{M}{k} \frac{\partial \Psi}{\partial x}\right)^{2}+\left(\frac{\partial \Psi}{\partial x}\right)^{2}+\left(\frac{\partial \Psi}{\partial y}\right)^{2}\right\} d y d t
$$

For the calculation of the energy flow one has to take the real part of the velocity potential because the energy is not a linear expression. Further one has much profit of the series expansion (1) because the functions $\cos n \pi y$ are orthogonal, and consequently all cross products vanish after integration over $y$. This means that there is no energy flow due to interaction of the waves.

The results of the calculations are as follows: for all waves with exponential decay as $|x| \rightarrow \infty$, that is, for waves with $n>N$, one obtains

$$
E_{T}=E_{1}+E_{2}=0 \text {. }
$$


This could be expected because otherwise one cannot see how the process can have only a periodic time dependence. This is a check for the correctness of the formulae. For $0<n \leqq N$ and $x>0$ one obtains

$$
\begin{aligned}
& E_{1}=-\frac{\rho \pi\left(k M-\sqrt{k^{2}-\beta^{2} n^{2} \pi^{2}}\right)\left(k-M \sqrt{k^{2}-\beta^{2} n^{2} \pi^{2}}\right)}{4 k \beta^{4}\left(k^{2}-\beta^{2} n^{2} \pi^{2}\right)}, \\
& E_{2}=\frac{\rho \pi M\left(k-M \sqrt{\left.k^{2}-\beta^{2} n^{2} \pi^{2}\right)^{2}}\right.}{4 k \beta^{4}\left(k^{2}-\beta^{2} n^{2} \pi^{2}\right)} \\
& E_{T}=E_{1}+E_{2}=\frac{\rho \pi\left(k-M \sqrt{k^{2}-\beta^{2} n^{2} \pi^{2}}\right)}{4 k \beta^{2} \sqrt{k^{2}-\beta^{2} n^{2} \pi^{2}}} .
\end{aligned}
$$

The factor $k-M \sqrt{k^{2}-\beta^{2} n^{2} \pi^{2}}$ is always positive for $M<1$. The factor $k M-\sqrt{k^{2}-\beta^{2} n^{2} \pi^{2}}$ is positive for $k / \pi<n<k /(\beta n)$. Hence the energy flow for the incoming waves is negative but $E_{T}$ is positive for all waves. For $n=0$ and $x>0$ one obtains

$$
E_{1}=\frac{\rho \pi(1-M)^{2}}{8 k \beta^{4}}, \quad E_{2}=\frac{\rho \pi M(1-M)^{2}}{8 k \beta^{4}}, \quad E_{T}=\frac{\rho \pi(1-M)}{8 k \beta^{2}} .
$$

All these quantities are positive because here we have always to do with an outgoing wave.

For $0<n \leqq N$ and $x<0$ the results are

$$
\begin{aligned}
& E_{1}=-\frac{\rho \pi\left(k M+\sqrt{k^{2}-\beta^{2} n^{2} \pi^{2}}\right)\left(k+M \sqrt{\left.k^{2}-\beta^{2} n^{2} \pi^{2}\right)}\right.}{4 k \beta^{4}\left(k^{2}-\beta^{2} n^{2} \pi^{2}\right)} \\
& E_{2}=\frac{\rho \pi M\left(k+M \sqrt{k^{2}-\beta^{2} n^{2} \pi^{2}}\right)^{2}}{4 k \beta^{4}\left(k^{2}-\beta^{2} n^{2} \pi^{2}\right)} \\
& E_{T}=-\frac{\rho \pi\left(k+M \sqrt{k^{2}-\beta^{2} n^{2} \pi^{2}}\right)}{4 k \beta^{2} \sqrt{k^{2}-\beta^{2} n^{2} \pi^{2}}}
\end{aligned}
$$

and for $n=0$ and $x<0$,

$$
E_{1}=-\frac{\rho \pi(1+M)^{2}}{8 k \beta^{4}}, \quad E_{2}=\frac{\rho \pi M(1+M)^{2}}{8 k \beta^{4}}, \quad E_{T}=-\frac{\rho \pi(1+M)}{8 k \beta^{2}} .
$$

Hence for $x<0$ we have $E_{1}<0$, which is in agreement with the fact that there exist only outgoing waves for $x<0$. The energy flow $E_{2}$ is again positive but $E_{T}$ is always negative. The conclusion is that we have upstream and downstream for all waves an energy transport in a direction away from the source.

The formulae for the problem with a circular cylindrical tube can be derived in the same way. The expressions for the energy flow in this case can be found by replacing everywhere in the formulae of the first problem $n$ by $\lambda_{n} / \pi$ and multiplying the righthand sides by $2 / J_{0}^{2}\left(\lambda_{n}\right)$. The expressionsfor $n=0$ follow in thiscasefrom the general formulae. Of course the conclusions for the energy flow are the same as above. This means that it is not possible to gain energy from infinity. 
Note: Details of the calculations can be found in [4]. In [4] it is also shown that when an integer $n$ exists with $k=n \pi \beta$, resp. $k=\lambda_{n} \beta$, then a steady state solution does not exist, but the velocity potential increases proportionally with $\sqrt{t}$ for $t \rightarrow \infty$.

\section{REFERENCES}

[1] P. le Grand, The screening of sound in a subsonic flow by a cylindrical airbubble layer and a semi-infinite tube. Thesis, University of Groningen, 1968.

[2] H. W. Hoogstraten and B. Kaper, Propagation of sound waves in a moving medium. Journal of Engineering Mathematics, Vol. 5, No. 4, October 1971, 295-305.

[3] P. A. Thompson, Compressible fluid dynamics. McGraw-Hill, 1971.

[4] P. le Grand, Geluidsgolven in golfgeleiders met een stromend medium. Memorandum Nr. 80, Dept. of Applied Mathematics, Technical University Twente, 1975. 